\title{
THE RESEARCH ON THE JUDGMENT OF PADDY RICE'S NITROGEN DEFICIENCY BASED ON IMAGE
}

\author{
Jun Sun ${ }^{1,2, *}$, Hanping Mao ${ }^{1}$, Yiqing Yang ${ }^{1}$ \\ ${ }^{1}$ Key Laboratory of Modern Agricultural Equipment and Technology, inistry of Education \& \\ Jiangsu Province, Jiangsu University, Zhenjiang, Jiangsu Province, China 212013 \\ ${ }^{2}$ School of Electrical and Information Engineering, Jiangsu university, Zhenjiang ,Jiangsu \\ Province, China 212013 \\ * Corresponding author, Address: School of Electrical and Information Engineering, Jiangsu \\ University, Zhenjiang 212013,Jiangsu Province,China, Tel:(0)13775544650, \\ Fax:051188780311,Email:sun2000jun@ujs.edu.cn
}

Abstract: Because of the unreliability judgment of paddy rice's nitrogen deficiency depending on the traditional artificial naked eye, in this article, the way of the paddy rice's nitrogen deficiency examination based on image is put forward, to achieve the precise fast lossless detection and judgment on the paddy rice's nitrogen. Based on the sorting function of SMV, paddy rice leaf's visible images are gathered, the texture features of image are extracted, the RBF nuclear function is chosen, the penalty coefficient $\mathrm{C}$ and the regularity coefficient $\gamma$ are set, and the SVM sorting model is constructed. The recurrence sentencing rate to the training sample achieves $100 \%$. The examination is caught on the test sample, and the accuracy rate of examination recognition achieve $95 \%$, which indicates that the method of paddy rice's nitrogen lossless examination judgment by image is effective and feasible to achieve the precise fast judgment on paddy rice's nitrogen.

Keywords: SVM (Support Vector Machine), paddy rice, image, nitrogen

\section{INTRODUCTION}

The nitrogen is one of the most major limit factors which affects the paddy rice growing, and it is the important ingredient which constitutes the

Please use the following format when citing this chapter:

Sun, J., Mao, H. and Yang, Y., 2009, in IFIP International Federation for Information Processing, Volume 294, Computer and Computing Technologies in Agriculture II, Volume 2, eds. D. Li, Z. Chunjiang, (Boston: Springer), pp. 1049-1054. 
crops organic matter. The lack of paddy rice's nitrogen nutrition directly influences the growing of paddy rice, and exerts an adverse influence to paddy rice's quality and the output. Therefore the prompt judgment of the paddy rice's nitrogen whether to lack is more and more needed in paddy rice's growth process. But the traditional naked eyes' judgment has the inevitable human factor. Therefore one intelligent examination judgment method is urgently needed. The lossless detection technology provides one new method for carrying on the rapid, accurate, non-destructive detection on the paddy rice crops nitrogen condition.

Support Vector Machine is the new generation machine learning algorithm which develops on the statistics theoretical basis. When it compares with the neural network method, this algorithm has the merits of the simply structure, the strong pan-ability and so on, and it has not the overstudy problem, specially aims at the finite sample situation, in order to get the optimal solution under the existing information. Support Vector Machine transforms the actual problem to the higher dimensional feature space through the nonlinear transformation, in the higher dimensional space, the structure of linear decision function realizes nonlinear decision function of original space decision function, which can guarantee that it has the better disseminate ability(Fang,2006). This article uses the SVM method of Support Vector Machine to set up the model and judge the paddy rice whether to lack nitrogen. At present, in domestic and foreign countries, the crops nitrogen nutrition lossless monitoring and the examination research mainly concentrate on the research of the leaf spectrum index and the remote sensing spectrum index. The literature report has not been seen that uses Support Vector Machine's method to conduct the research to the paddy rice's nitrogen deficiency detection based on the image characteristic, so this article research has the significance.

\section{PADDY RICE CULTIVATION EXPERIMENT}

The experimental objects are the paddy rice seedlings which come from Zhenjiang suburb farmland. The paddy rice variety is new fragrant superior 80 , and the growing cycle is 115 days. The testing site is the big intelligence control greenhouse vent in the Jiangsu University. On August 1, 2007, they are transplanted using the water cultivation method, and the standard nourishment liquid formulation (except the quantity of nitrogen) is compound according to the international paddy rice nourishment liquid formulation. The paddy rice is fixed in the experimental cylinder with the sponge. Row with line distance is $0.3 \mathrm{~m} \times 0.3 \mathrm{~m}$, and each cylinder is a plot.

In order to gain different nitrogen-containing level of the paddy rice leaf blade, in view of nitrogen nutrition in the paddy rice nourishment liquid, it 
can be divided into 3 levels N1, N2, N3, that is to say, the nitrogen normal $(40 \mathrm{mg} / \mathrm{L})$, the nitrogen lacking $(0.5 \times 40 \mathrm{mg} / \mathrm{L})$, the nitrogen lacking $(0.25 \times 40$ $\mathrm{mg} / \mathrm{L}$ ), and 4 times repetition are set. Each cylinder has 4 clumps, and at average level each clump has the paddy rice plant number of 2.5. Each cylinder installs 10L nourishment liquid, it examines one time a weak, and the nourishment liquid is replaced each time for every 30 days.

The normal nitrogen-containing sample comes from the N1 level sample, and the nitrogen deficiency sample comes from the N2 N3 levels sample.

\section{GATHERING IMAGE CHARACTERISTIC OF PADDY RICE LEAF}

The paddy rice leaf's images are gathered on the scene using the high speed image gathering system. To avoid the paddy rice leaf complex background effect, after being picked the paddy rice leaf in the experimental field, it is immediately placed on the experiment table's white paper in the dark awning carries on the image photography. An example image is shown in Figure 1.

The paddy rice leaf image's characteristics are mainly the texture features, color greyscale characteristic and so on. The extraction image characteristic must avoid the object's shape and size to affect the effectiveness of the extraction characteristic.

Fig. 1 paddy rice leaf's original chart

The paddy rice leaf image is processed by filtering the background having independence on leaf. The color characteristics of paddy rice leaf are red $(\mathrm{R})$, blue $(\mathrm{B})$, green $(\mathrm{G})$, chroma $(\mathrm{H})$, light intensity (I) and saturation $(\mathrm{S})$ value .The other color parameter, such as $\mathrm{R} /(\mathrm{G}+\mathrm{B}), \mathrm{G} /(\mathrm{R}+\mathrm{B}), \mathrm{B}(\mathrm{R}+$ G) , G/R, G/B and R/B, can be caught out by Excel software. Researches indicate that paddy rice leaf change obviously with the inner nitrogen change. The red light and green light increase with the nitrogen content rise. The blue light decreases with nitrogen content increasing. Color parameter $\mathrm{R}, \mathrm{G}$, $\mathrm{G} /(\mathrm{R}+\mathrm{B}), \mathrm{B} /(\mathrm{R}+\mathrm{G}), \mathrm{G} / \mathrm{B}, \mathrm{R} / \mathrm{B}$ have relation with the nitrogen content, so they can be taken as the parameter on which nitrogen diagnostic based(Xue ,2002;Zhang, 2003;Lu,2000). 


\section{SVM PATTERN RECOGNITION METHOD}

Suppose $\mathrm{m}$ dimensions training sample input data $\mathrm{xi}(\mathrm{i}=1,2,3 \ldots \mathrm{M})$ belonging to the category 1 or the category 2 respectively. When the training data has the noise, the feature space cannot be separated linearly generally. In order to permit the indivisibility, it introduces nonnegative relaxation variable $\xi_{i}$ :

$$
y_{i}\left(W^{T} x_{i}+b\right) \geq 1-\xi_{i}, i=1,2, \ldots M
$$

The combination optimization question becomes:

$$
\min Q(W, b, \xi)=\frac{1}{2}\|W\|^{2}+C \sum_{i=1}^{M} \xi_{i}^{p}
$$

The constraint condition is:

$$
y_{i}\left(W^{T} x_{i}+b\right) \geq 1-\xi_{i} \mathrm{i}=1, \ldots \mathrm{M}
$$

In the formula $\xi=\left(\xi_{1}, \ldots \xi_{M}\right)^{T}, \mathrm{C}$ is the marginal coefficient, $\mathrm{P}$ is a norm. Introducing the nonnegative Lagrange multiplier $\alpha_{i}$ with $\beta_{i}$, may result in

$$
Q(W, b, \xi, \alpha, \beta)=\frac{1}{2}\|W\|^{2}+C \sum_{i=1}^{M} \xi_{i}-\sum_{i=1}^{M} \alpha_{i}\left[y_{i}\left(W^{T} x_{i}+b\right)-1+\xi_{i}\right]-\sum_{i=1}^{M} \beta_{i} \xi_{i}
$$

The above formula suppose various variables' deflection is equal to 0 , and may obtain the corresponding dual question when it substitutes in the above formula.

$$
\max Q(\alpha)=\sum_{i=1}^{M} \alpha_{i}-\frac{1}{2} \sum_{i, j=1}^{M} \alpha_{i} \alpha_{j} y_{i} y_{j} K(x, y)
$$

The constraint condition is

$$
\sum_{i=1}^{M} y_{i} \alpha_{i}=0 \quad C \geq \alpha_{i} \geq 0 \quad i=1, \ldots M
$$

And $\mathrm{K}(\mathrm{x}, \mathrm{y})$ is the nuclear function.

The optimal sorting decision function is:

$$
D(x)=\sum_{i \in S} \alpha_{i} y_{i} x_{i}^{T} x+b
$$

To the non-linear problem, it can transform the non-linear problem through the nuclear function as the linear question in the higher dimensional space. And then the optimal sorting surface is found in the transformation space(Zhao,2007;Ge,2007). The sorting function becomes:

$$
D(x)=\sum_{i \in S} \alpha_{i} y_{i} K\left(x_{i}, x\right)+b
$$

The diagnosis method is:

$$
\begin{cases}\text { Normal } & w \text { henD }(x)>0 \\ \text { nitrogen deficiency } & \text { whenD }(x)<0\end{cases}
$$




\section{MODEL ESTABLISHMENT AND EXPERIMENT}

The establishment of recognition model must solve the question of choosing the nuclear function at first, according to the literature, the sorting question can be obtained a better result by using the radial base function. The radial base function can insinuate the nonlinear sample data to the higher dimensional feature space, process the sample data which has the nonlinear relations.

In this experiment the situation of the computer used is: Processor CPU1.40GB, 1G memory, XP operating system, Matlab7.0 edition. The number of paddy rice leaf's images which have been gathered in the research is 200 , taking 150 of them as training sample, 50 of them as test sample.

The sample import value are color parameters $R, G, G /(R+B), B /(R$ $+\mathrm{G}), \mathrm{G} / \mathrm{B}, \mathrm{R} / \mathrm{B}$, Output value is $\mathrm{D}$. D value is 1 when the nitrogen content is normal and $\mathrm{D}$ value is 0 when the nitrogen content is lack.

After determining the nuclear function, it needs carry on the optimization to the nuclear function parameter. The radial base function needs take definite parameter as penalty coefficient $\mathrm{C}$ and regularity coefficient $\gamma$. In this article, the different combinations are attempted and the results obtained are shown in Table 1. At last it determines $\mathrm{C}=3, \gamma=15$, the accuracy recurrence sentencing rate is $100 \%$, and the accuracy forecast rate is $95 \%$.With the traditional BP neural network contrast, the input layer node number of BP Network selected is 7 , and the implicit layer node number is 10 , the output layer node number is 1 . The recognition results using the SVM algorithm and the BP neural network are shown in Table 2.

Table 1.Different parameter choice and test result

\begin{tabular}{lcccc}
\hline C & Gamma & $\begin{array}{c}\text { Support vector } \\
\text { number }\end{array}$ & $\begin{array}{c}\text { the accuracy recurrence } \\
\text { sentencing rate }\end{array}$ & The accuracy forecast rate \\
\hline 1 & 1 & 34 & $80 \%$ & $70 \%$ \\
3 & 15 & 35 & $100 \%$ & $95 \%$ \\
5 & 20 & 40 & $90 \%$ & $85 \%$ \\
10 & 25 & 40 & $80 \%$ & $75 \%$ \\
20 & 30 & 43 & $80 \%$ & $75 \%$ \\
\hline
\end{tabular}

Table.2 Training times and test result reference table

\begin{tabular}{lccc}
\hline Algorithm & Training time $(\mathrm{ms})$ & Test sample number & Correct judgment rate \\
\hline SVM & 51 & 50 & $95 \%$ \\
BP Network & 1115 & 50 & $85 \%$ \\
\hline
\end{tabular}




\section{CONCLUSION}

In the recent years, the lossless detection technology has been obtained the widespread attention in the crops nitrogen nutrition diagnosis and the nitrogen fertilizer recommendation. In this article, Support Vector Machine is applied in paddy rice whether to lack nitrogen judgment research in the first time, and the very good judgment results are achieved. The test result has indicated that the SVM algorithm has a big enhancement compared to the traditional BP neural network from the training time and the correct recognition rate, it provides one new method for the nitrogen deficiency judgment of other plants, and it has certain promoted significance.

\section{ACKNOWLEDGEMENTS}

Funding for this research was provided by China Postdoctoral Science Foundation under Grant (NO:20070420972); Jiangsu University High-grade Specialty Person Scientific Research Foundation under Grant (NO: 05JDG050); “863"Project(2008AA10Z204). The first author is grateful to the Jiangsu University for providing him with pursuing a postdoctoral degree.

\section{REFERENCES}

Fang Ruiming. Induction Machine Rotor Diagnosis using Support Vector Machines and Rough Set[J]. Lectures notes on Artificial Intelligence,2006.Vol: 631 637(in Chinese)

Ge Guangying. Algorithm of vehicle detection and pattern recognition using SVM. Computer Engineering. 2007(6):6-10(in Chinese)

Lu Renfu,Daniel E Guyer, Randolph M Beaudry. Determination of firmness and sugar content of apples using near-infrared diffuse reflectance. Journal of Texture Studies,2000,31:615- 630(in Chinese)

$\mathrm{Xu}$ Guili, Mao Hanping, Li Pingping. Extracting Color Features of Leaf Color Images. Transactions of the CSAE. 2002,7:150-154(in Chinese)

$\mathrm{Xu}$ Guili,MAO Hanping,LI Pingping. Application Algorithm to Extract Color Images Color and Textures Features. Computer Engineering. 2002,6:25-27(in Chinese)

Zhang Wei,Mao Hanping,LI Pingping, XIA Zhijun. Research on Extracting Color and Texture Features of Plant Nutrient Deficiency Leaves' Image. Journal of Agricultural Mechanization Research. 2003,4:60-63(in Chinese)

Zhao Jiewen,Hu Huaiping,Zou Xiaobo.Application of support vector machine to apple classification with near-infrared spectroscopy. Transactions of the CSAE.2007, 23(4):149-152.(in Chinese) 